\title{
Distributed Coordination and Control of Formation Flying Spacecraft
}

\author{
Michael Tillerson, Louis Breger, and Jonathan P. How \\ MIT Department of Aeronautics and Astronautics \\ \{mike_t, lbreger, jhow\}@mit.edu
}

\begin{abstract}
Formation flying is a key technology for many planned space missions that will use multiple spacecraft to perform distributed observations. This paper extends previous work on the design of a highly distributed formation flying control system that uses linear programming to determine minimum fuel trajectories for the spacecraft to remain within some specified tolerance of their "desired points". The primary contribution of this paper is that it presents a direct procedure for calculating the fleet reference point (called the virtual center) that can be used to determine the desired states for each vehicle in the fleet. The calculation of this virtual center is based on measurements available from the relative navigation sensing system (carrier-phase differential GPS) developed for this application. The selection of the reference point includes a weighting on fuel use across the fleet, which facilitates increased coordination and cooperation within the decentralized control system. Full nonlinear simulations are presented to demonstrate the reduction in fuel use that can be obtained with this improved cooperation.
\end{abstract}

\section{Introduction}

A large number of future planned space missions are based on a new paradigm that will use coordinated formation flying microsatellites to provide flexible, lowcost access to space [1]. The goal of spacecraft formation flying is to maintain a fleet of vehicles in a desired relative geometry over extended periods of time while minimizing fuel cost. Three main issues to be addressed in the formation flying control problem are the selection of the: (i) Reference point for the fleet; (ii) Dynamics for determining the desired state for each vehicle; and (iii) Dynamics used to represent the motion of spacecraft relative to the desired state in the controller.

Three methods of specifying the reference point are considered in this paper. The first is a point on a reference orbit that is propagated with the fleet. The second is the traditional leader-follower, where a leader is the formation reference point. The third approach 0-7803-7896-2/03/\$17.00 @2003 IEEE involves a virtual center calculation, which uses measured state information from the vehicles in the fleet to calculate the location of the virtual center of the fleet that minimizes all vehicles' state errors. The virtual center approach is similar to the formation feedback method presented in Ref. [2], but is applied to spacecraft formation flying in low earth orbit and uses a fuel weighting method to equalize fuel use across the fleet. Another distinction is that the calculation of the virtual center is based on measurements available from the relative navigation estimator developed for this application $[3,4]$. Using the virtual center extends the previous formation-keeping control in [5] to formation flying control by enabling extensive cooperation between the vehicles.

Desired states relative to the reference point are specified using passive apertures designed with the closedform solutions to various linearized equations of relative motion $[6,7,8]$. Passive apertures are designed to result in drift-free motion, but disturbances such as differential drag cause the formation to disperse, necessitating feedback control. This paper uses a control algorithm based on linear programming (LP) to minimize fuel cost [5]. Combining the reference point calculation (virtual center) based on the CDGPS measurements with the decentralized LP control, this paper presents solutions to each of the three main questions in the formation flying control problem.

\section{Control Formulation}

The LP trajectory planner was presented in detail in Ref. $[5,10]$. The form of the LP is

$$
\min \|u\|_{1} \text { subject to } \mathbf{A} u \leq \mathbf{b}
$$

where $u$ is the vector of fuel inputs $(\Delta V)$ at each time step and $\mathbf{A}, \mathbf{b}$ are functions of the linearized spacecraft dynamics, initial conditions, and final conditions. The LP determines the control inputs for a specified time interval that minimizes the fuel cost while satisfying the constraints on the trajectory.

One of the first steps in applying the LP technique for a spacecraft control system is to determine the desired Proceedings of the American Control Conference
Denver, Colorado June $4-6,2003$ 


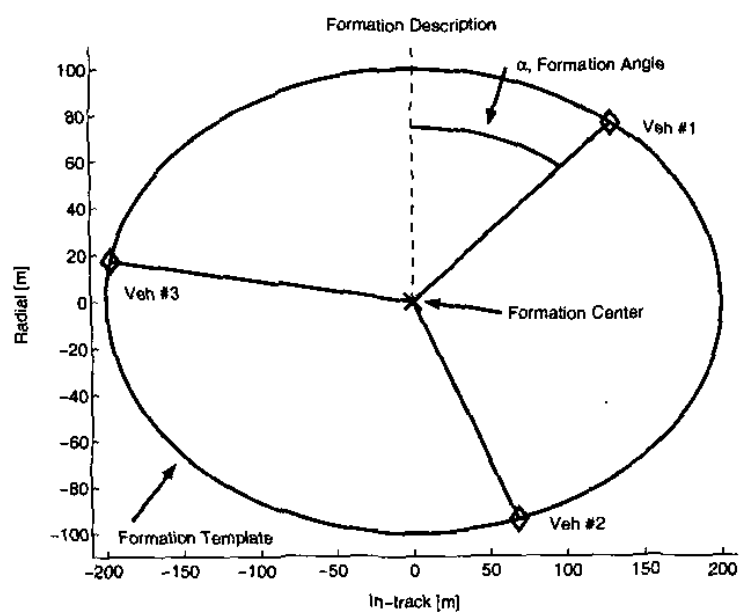

Fig. 1: The formation geometry is described relative to the formation center, marked by a $\times$. Each vehicle state is specified by a radius and an angle.

state. An error box is fixed to the desired state to provide a position tolerance for the satellite. A key point in this work is that the error box is specified relative to a desired point for the spacecraft. The primary purpose of this paper is to demonstrate how these desired points can be specified for the formation, and how the error of the spacecraft from the desired point can be estimated using the onboard carrier-phase differential GPS (CDGPS) system [4].

\section{Reference Point Coordination}

The formation-keeping LP algorithm in Sec. 2 is formulated to control a single spacecraft to maintain a desired state to within some tolerance specified by an error box centered on the desired state. The formationkeeping algorithm is applied independently to each spacecraft, allowing distribution of the required computation across the fleet. The desired state is specified relative to a reference point, which can be chosen to enable cooperation between the spacecraft in the fleet, enabling formation flying.

Figure 1 depicts a typical scenario for a formation of three spacecraft. The formation radius defines the passive aperture size. The formation angle is measured from maximum positive radial displacement. The initial conditions and closed-form solutions to the relative dynamics are used (with the drift free constraints imposed [8]) to find the desired states at future times. The desired state is specified relative to the formation center, which is determined relative to a formation reference point. Three methods for determining the reference point are discussed in the following subsections. Each method is evaluated for its complexity and the amount of information flow required for its execution.

\subsection{Reference Orbit}

A simple method of specifying the reference point is using the reference orbit. The reference orbit is a point

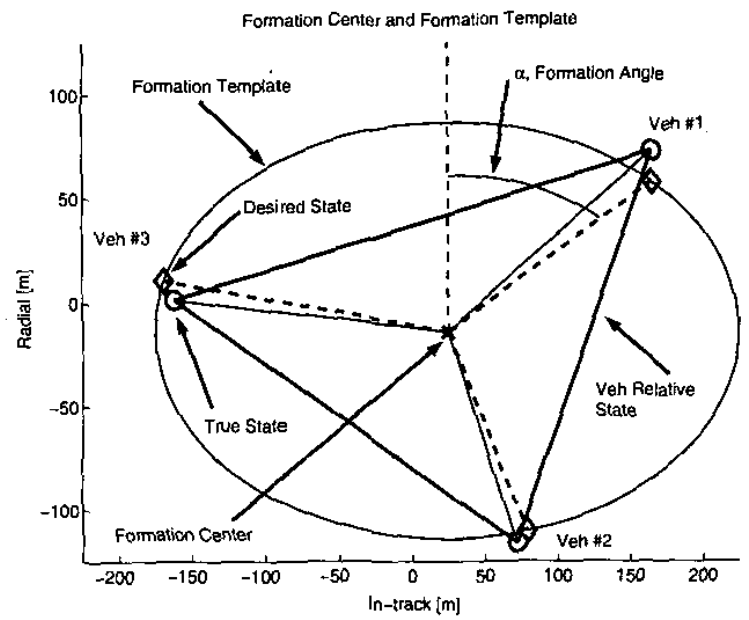

Fig. 2: Virtual center calculated from measured relative states (thick solid) and used to determine desired states (dashed) and actual state (thin solid) of each spacecraft.

in space that is propagated using a model that describes the average fleet orbit. The formation center is attached to the reference orbit and is used to specify the desired spacecraft states. The reference point is described by the nonlinear orbit equations, requiring little communication between vehicles. Also note that the reference point is not specified using measurements, so there is no uncertainty in the state due to sensor noise. A disadvantage of this approach is that the reference point does not naturally experience the fleet disturbances. Instead, a disturbance model must be included in the propagation. If the model is inaccurate, the fleet will track a reference orbit that does not describe the fleet motion. Instead of using control effort to maintain the fleet, effort would be wasted "chasing" a mathematical point in space that does not move with the fleet.

\subsection{Leader-Follower}

Another common method of specifying the reference point is to let a vehicle be the leader and fix the reference point to the leader spacecraft. The advantage is that the reference point is on a spacecraft, which eliminates the need to propagate the motion and it naturally captures the absolute disturbances. The leader-follower method requires little information flow, because the reference state is just the state of the leader spacecraft.

One leader-follower configuration places the leader at the aperture center, but this makes it prohibitively expensive to change leaders in the formation. Alternately, the leader spacecraft could be one of the vehicles on the passive aperture. The desired state for each follower spacecraft then becomes the desired state for the follower relative to the aperture center minus the desired state of the leader from the aperture center. This simplifies the transition between leaders, because no maneuvers are required. However, instantaneously switch- 
ing leaders could cause a jump in the desired state of each spacecraft and must be done with care.

A disadvantage of this method is that the leader does not represent the average fleet motion, forcing some followers to overcome larger disturbances than others. Also, the leader spacecraft will use minimal fuel, because its state never experiences error. To equalize control effort across the fleet, the leader spacecraft can be alternated based on the fuel usage/status within the fleet.

\subsection{Virtual Center}

A third approach is to use a "virtual center" as the reference point. The reference state in this case is estimated using measurements between the spacecraft in the fleet. An advantage of the virtual center is that it represents the weighted average motion of the fleet, including an average of the actual disturbances. The weighted average enables cooperation within the fleet. The virtual center method presented here is similar to the formation feedback method for multiple vehicle control presented in Ref. [2], but our approach differs, because we show how the virtual center can be implemented using sensors planned for formation flying missions $[3,4]$. The navigation algorithm presented in Ref. [3] uses decentralized estimators to filter the CDGPS measurements, precisely determining the location of each spacecraft relative to a "reference" vehicle. In the following discussion, we shall assume that the reference vehicle is the leader, although that is not necessary in general. Given the estimated states relative to the leader, it is possible to precisely determine the formation center.

Fig. 2 shows a formation of three spacecraft. The thick solid lines are known or measurable distances and the thin solid lines represent the true distances to the virtual center that are compared to the specified desired state relative to the virtual center (dashed lines). To calculate the relative position and velocity of the center, a measurement reference must be specified. In the figure, the reference frame is attached to spacecraft \#1, which will be referred to as the reference spacecraft. Inter-spacecraft states, $\vec{x}_{1 i}$, are measured relative to the reference spacecraft, represented by the solid lines in Fig. 2. The virtual center state, $\vec{x}_{c}$, is also specified relative to the reference spacecraft. Each spacecraft state relative to the virtual center is

$$
\vec{x}_{c i}=\vec{x}_{1 i}-\vec{x}_{c}
$$

The error states are the difference between the state of each spacecraft relative to the center, $x_{c i}$, and the desired state for that spacecraft, which is also specified relative to the center. Error states in the figure are the differences between the $\diamond$ and $\circ$ for each spacecraft.

$$
\vec{x}_{c i}-\vec{x}_{i, \text { des }}=\vec{e}_{i}
$$

Substituting Eq. 2 for $x_{c i}$ yields the following expression for the vehicle error in terms of known quantities and the unknown virtual center, $\vec{x}_{c}$,

$$
\vec{x}_{1 i}-\vec{x}_{c}-\vec{x}_{i, d e s}=\vec{e}_{i}
$$

The error equation for each spacecraft becomes

$$
\begin{aligned}
& {\left[\begin{array}{c}
\vec{x}_{11}-\vec{x}_{1, \text { des }} \\
\vec{x}_{12}-\vec{x}_{2, \text { des }} \\
\vdots \\
\vec{x}_{1 N}-\vec{x}_{N, \text { des }}
\end{array}\right]-\left[\begin{array}{c}
I \\
I \\
\vdots \\
I
\end{array}\right]\left[\vec{x}_{c}\right]=\left[\begin{array}{c}
\vec{e}_{1} \\
\vec{e}_{2} \\
\vdots \\
\vec{e}_{N}
\end{array}\right] \equiv \vec{e}} \\
& \text { or } b-A x=\vec{e}
\end{aligned}
$$

The virtual center location $\vec{x}_{c}$, is chosen to minimize the sum of the errors, $\|\vec{e}\|_{2}=(b-A x)^{T}(b-A x)$. A weighting matrix, $W$, can be included to increase the importance of a vehicle or state, giving the weighted least-squares problem $\min (b-A x)^{T} W(b-A x)$, with solution

$$
\hat{x}_{c}=\left(A^{T} W A\right)^{-1} A^{T} W b
$$

Using current fuel use in the weighting matrix allows fuel use across the fleet to be equalized over time.

Using this method, updates can be made to the virtual center state every time step or periodically with a propagation of the virtual state between updates. A key advantage of this method is that the disturbances affecting each spacecraft become differential disturbances relative to the fleet average, which will lower fuel costs. Measuring spacecraft error relative to a regularly updating virtual center makes the absolute motion of the fleet unobservable to individual spacecraft. Thus, absolute motion will not enter into the LP, ensuring control effort is only utilized for relative geometry maintenance. A disadvantage is that the virtual center calculation must be centralized, since the current and desired states of all spacecraft must be collected in one place to find the virtual center, requiring an increase in communication throughout the fleet. Also, noise and uncertainty in measurements will lead to uncertainty in the virtual center state.

A further issue with this approach is that the virtual center is a function of the states of all the vehicles in the fleet, so any control effort by one vehicle will influence all of the other vehicles. When a vehicle uses a control input to correct an error, the control input assumes the virtual center is fixed over the plan horizon. However, the location of the center will change over time as each vehicle moves. The control inputs from the other vehicles can be included in this decentralized control algorithm by having all vehicles "publish" a list of planned control actions and then having each vehicle include the inputs of the other vehicles as disturbance inputs into their dynamics. The control inputs get scaled to give the motion for the virtual center in the near future. Unfortunately, there is no guarantee
Proceedings of the American Control Conference Denver, Colorado June 4-6, 2003 
the published plans will get fully implemented, which may cause errors in the trajectory design.

Another way to predict the effect of external control inputs on the virtual center is to form a centralized LP to solve for all vehicles' control inputs simultaneously. The virtual center state at each time step is described in terms of the vehicle states, as in Eq. 6, capturing the center motion due to all control inputs. Control input solutions and trajectories would then have to be sent to each vehicle, increasing the communication load, thereby making this approach intractable for larger fleets.

\section{Simulations Results}

Several simulations were performed to demonstrate the effectiveness of the control system presented in this paper. FreeFlyer ${ }^{\mathrm{TM}}$ orbit simulation software is used as the nonlinear propagator for each satellite while MATLAB ${ }^{\mathrm{TM}}$ mathematical software is used to implement the controller. The entire control system is executed without human intervention.

The simulation consists of three vehicles, each modeled as a $45 \mathrm{~kg}$ Orion spacecraft [3] with slightly different drag coefficients. Other disturbances, such as gravity perturbations, solar radiation pressure, atmospheric lift, and third body effects are activated in the FreeFlyer ${ }^{\mathrm{TM}}$ propagator. Sensor noise is included in the simulation as a white noise component added to the true relative state. The magnitude of the sensor noise is kept less than $2 \mathrm{~cm}$ for position and $0.5 \mathrm{~mm} / \mathrm{s}$ for velocity, based on expected CDGPS sensor noise [3]. Spacecraft thrusters provide a maximum acceleration of $0.003 \mathrm{~m} / \mathrm{s}^{2}$, which corresponds to thrusting for a full time-step. The formation is initialized on a reference orbit (semi-major axis $6900 \mathrm{~km}$, eccentricity of $0.005)$ similar to a space shuttle orbit. The reference orbit inclination is $35^{\circ}$, introducing significant differential gravity disturbances for spacecraft with inclination differences. See Ref. [11] for the full details on the simulation parameters.

When using the virtual center procedure, the reference point is updated at every time-step. The relative dynamics are discretized on a 10.8 second time interval to match the propagation step-size. Formation flying problems are planned over a half-orbit time horizon. The LP formation flying formulation restricts control inputs and applies position constraints to every sixth time-step [10], which reduces LP solution times to about $1-3$ seconds. The robust LP approach in Ref. [9] is used to account for sensor noise and the always feasible approach in Ref. [10] is also used. The error box size for position tolerance is $10 \mathrm{~m}$ in-track, $5 \mathrm{~m}$ radial, and $5 \mathrm{~m}$ cross-track, which meets the tolerance requirement of $10 \%$ of the baseline for all formations in the simulation (baseline tolerance for TechSat21).

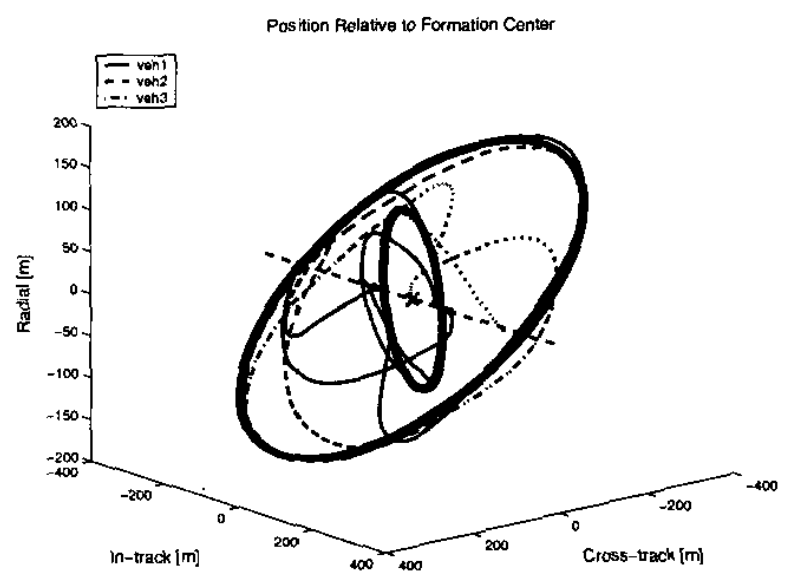

Fig. 3: Relative motion for 3 vehicle formation. Sequence is in-track separation, small ellipse, larger ellipse, in-track separation.

The simulation contains three formation maneuvers with formation flying at each configuration. The spacecraft paths during maneuvers are shown with respect to the virtual reference point in Figure 3. The formation begins and ends in similar in-track separations. Two passive aperture formations are maintained for approximately seven days each to observe any long and short term effects of the disturbances, particularly the gravity perturbation effects. The first aperture projects a $400 \times 200 \mathrm{~m}$ ellipse in the in-track-radial plane and a circle with a $100 \mathrm{~m}$ radius in the radial-cross-track plane. The second aperture projects a $600 \times 300 \mathrm{~m}$ ellipse in the in-track-radial plane and a $300 \mathrm{~m}$ radius circle in the in-track-cross-track plane. Aperture position assignment is coordinated through the procedure described in Ref. [5] with a plan horizon of one orbit. This simulation successfully demonstrates the control system presented in this paper for all aspects of a spacecraft formation flying mission.

\subsection{Analysis of Controller Performance}

Full simulation fuel costs for the leader-follower and fuel-weighted virtual center methods are shown in Figs. 4 and 5 . The fuel cost figures show three reconfiguration maneuvers, each of which uses a significant amount of fuel over a short period of time. The longer, constant slope segments correspond to the periods of formation-flying. Comparing the two figures, it is clear that the Leader-Follower method has a higher fuel cost than the virtual center method throughout the mission. In the leader-follower figure, a spacecraft exerting no control (the flat lines) is presently the leader of the formation and, consequently, has no state error. When the total control effort exerted by one spacecraft significantly exceeds that of the other spacecraft, the leader is switched to balance overall fuel use. In comparison, the fuel-weighted virtual center method spreads the error out amongst all of the spacecraft, with the objective of placing the virtual center in such a way as Proceedings of the American Control Conference 
Table 1: Table of fuel costs for the virtual center simulations. The simulation number corresponds to the level of coordination in the controller for each simulation. The spacecraft is indicated by SC\#. The maneuver types are followed by the number of orbits the maneuver was performed for. FF indicates formation flying and FM represents formation maneuvers.

\begin{tabular}{|l||c|c|c||c|c|c||c|c|c|}
\hline \multicolumn{1}{|c||}{} & \multicolumn{3}{c||}{ Sim 1 } & \multicolumn{3}{c||}{ Sim 2 } & \multicolumn{3}{c|}{ Sim 3 } \\
\cline { 2 - 11 } Maneuver Type & SC 1 & SC 2 & SC 3 & SC 1 & SC 2 & SC 3 & SC 1 & SC 2 & SC 3 \\
\hline FF \#1 (4) mm/s/orbit & 0.509 & 0 & 0.523 & 0.652 & 0 & 0.112 & 0.542 & 0 & 0.341 \\
\hline FM \#1 (1) mm/s & 163 & 150 & 171 & 160 & 148 & 168 & 169 & 157 & 165 \\
\hline FF \#2 (101) mm/s/orbit & 3.07 & 2.82 & 2.43 & 2.70 & 2.54 & 2.26 & 2.39 & 2.48 & 2.51 \\
\hline FM \#2 (1) mm/s & 315 & 291 & 315 & 339 & 291 & 320 & 275 & 306 & 314 \\
\hline FF \#3 (90) mm/s/orbit & 8.14 & 6.90 & 6.18 & 7.42 & 6.57 & 6.59 & 6.59 & 6.73 & 6.82 \\
\hline FM \#3 (1) mm/s & 415 & 440 & 391 & 408 & 410 & 374 & 404 & 392 & 393 \\
\hline FF \#4 (14) mm/s/orbit & 2.64 & 4.69 & 2.30 & 1.71 & 0.696 & 1.45 & 3.90 & 2.25 & 0.541 \\
\hline \hline FM Total mm/s (3) & 893 & 881 & 877 & 907 & 849 & 862 & 848 & 855 & 872 \\
\hline FF Total mm/s (209) & 1141 & 1040 & 899 & 1019 & 920 & 931 & 979 & 1004 & 964 \\
\hline Total Fuel mm/s (212) & 2034 & 1921 & 1776 & 1926 & 1769 & 1793 & 1827 & 1859 & 1836 \\
\hline
\end{tabular}

to minimize global control effort across the formation. As a result, there is a nonzero fuel cost for all three spacecraft during the formation-flying mode.

The simulations using a virtual center reference point were performed for three different levels of fleet cooperation. The first simulation calculates the virtual center in the formation with equal weights on each vehicle in the fleet. The second simulation includes the control actions of other spacecraft in the control determination. The third simulation includes the external control inputs, as in the second simulation, and also adjusts the weighting of the vehicles based on fuel use. All three methods successfully achieve and maintain the specified configurations during the simulation. In the formation-flying mode, the vehicles are maintained approximately within the specified position tolerance due to the always-feasible formulation. The maximum deviation from the desired state for any simulation was less than $11 \mathrm{~m}$ in-track, $5 \mathrm{~m}$ radial, and $7 \mathrm{~m}$ cross-track. The total fuel cost data for each of the simulations is contained in Table 1.

\subsection{Formation Flying Analysis - FM}

The results in Table 1 show that there is no appreciable difference in fuel cost between the three different controllers for the formation maneuvers; however, this is not unexpected. The difference between the first two simulations is the inclusion of the control inputs of other vehicles in the low-level controller for formationflying. Therefore, there is no expected improvement in the formation maneuvers from this change. The last simulation adds fuel weighting to the calculation of the formation center. The fuel weighting is only updated once every two orbits, whereas the formation maneuvers occur over a single orbit. Some benefit can be expected, because the fuel weighting will reduce the differential disturbances of vehicles that have used large amounts of fuel, however, this change will be minimal over the course of one orbit.

\subsection{Formation Flying Analysis - FF}

For the two passive aperture formation flying maneuvers, the rate at which fuel is expended for each vehicle is heavily dependent on the cross-track disturbance. The in-track and radial control efforts are approximately equal for each vehicle in the formation, regardless of the spacecraft location in the aperture; however, the cross-track fuel use varies significantly for each vehicle. The cross-track disturbance results in a secular increase in the amplitude of the cross-track oscillatory motion, and the magnitude of this increase depends on the cross-track phasing. With a three vehicle formation, it is impossible to eliminate the disturbance completely for every vehicle, therefore, at least two vehicles will experience a cross-track disturbance and will expend more control effort than the other in response to the cross-track disturbance. Altering the phasing over time can equalize the average cross-track disturbance for all vehicles [12]. This method could be included in the control system presented in this paper, but note that the coordinated virtual center equalizes the fuel cost due to the cross-track disturbance through the calculation of the fuel-weighted virtual center.

\subsection{Total Fuel Cost Analysis}

The controllers can be compared by the total fuel cost for the mission. If a formation flying mission requires the entire fleet to perform the science observations, then the mission life will be limited by the vehicle with the greatest fuel use. The fuel expenditure for each vehicle during the mission is summarized in the last row of Table 1. The results show that the maximum fuel cost is reduced from $2.03 \mathrm{~m} / \mathrm{s}$ for the first simulation to $1.93 \mathrm{~m} / \mathrm{s}$ for the second simulation. However, the fuel cost for one vehicle is much larger than for the other two vehicles in both simulations. The third simulation utilizes the fuel weighting scheme to reduce the maximum fuel use by shifting control effort to the lower fuel cost vehicles. The result is a reduction in the maximum fuel cost to $1.86 \mathrm{~m} /$ sroceedings of the American Control Conference $_{\text {f }}$ Denver, Colorado June 4-6, 2003 


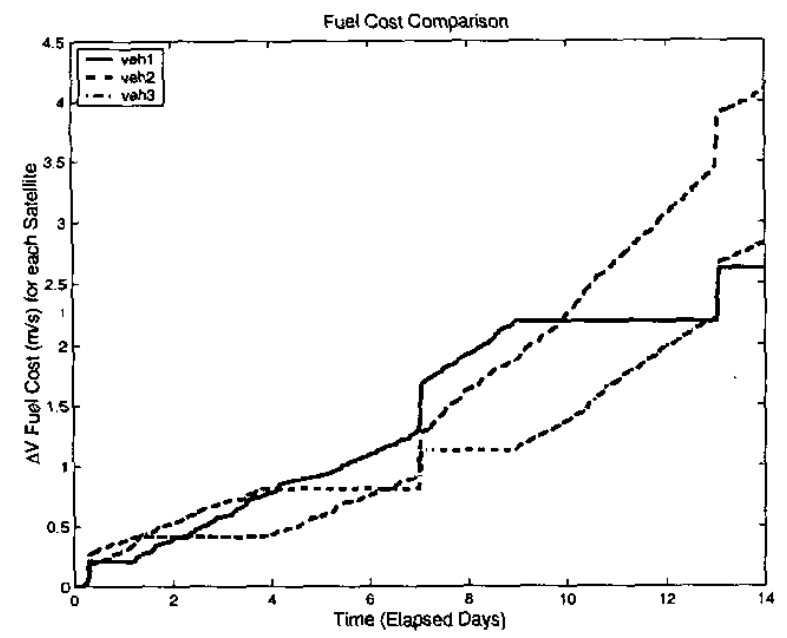

Fig. 4: $\Delta V$ 's for each vehicle, using Leader-Follower sharp rises indicate formation maneuvers and constant slope parts correspond to formation flying.

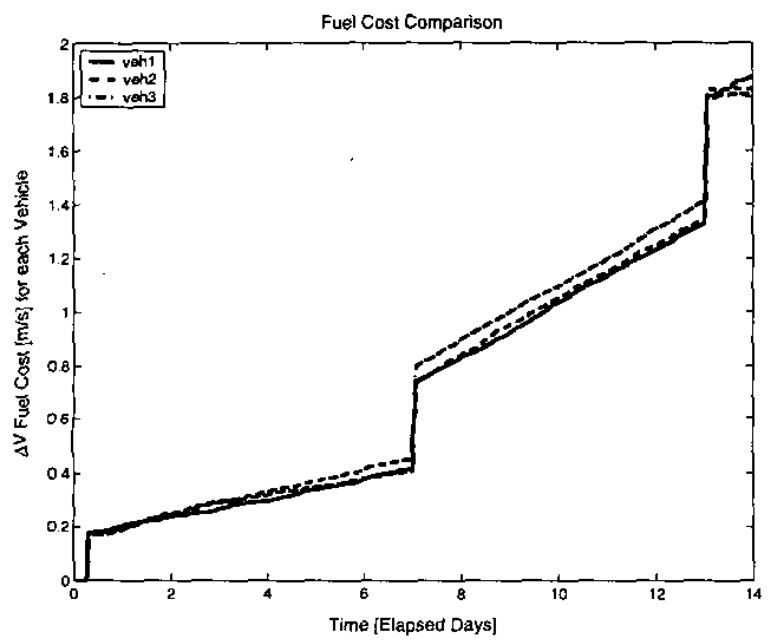

Fig. 5: $\Delta V$ 's for each vehicle, using Virtual Center sharp rises indicate formation maneuvers and constant slope parts correspond to formation flying.

\section{Conclusions}

This paper addresses the three main issues of the formation flying control problem: the reference point for the formation, the specification of the desired state, and the control to achieve or maintain the desired state. The primary contribution of this paper is that it presents a procedure for calculating the reference point (called the virtual center) for the fleet from which the desired states for each spacecraft can be readily calculated. Note that the calculation of this virtual center is closely tied to the planned formation flying sensor (CDGPS in LEO). The selection of the location of the center also includes a weighting on fuel use across the fleet, which facilitates increased coordination and cooperation within the decentralized control system. The result is an efficient real-time control system using the benefits of a fuel-optimal controller to plan control ac- tions and coordination between the fleet to further reduce fuel effort. The simulation results indicate that this control system can adequately maintain a formation at a fuel cost of $2-8 \mathrm{~mm} / \mathrm{s}$ per orbit. The simulations also clearly show that the virtual center approach required significantly less fuel than the leader-follower technique.

\section{Acknowledgments}

Research funded under NASA Grant \#NAG5-10440.

\section{References}

[1] J. Leitner, F. Bauer, D. Folta, M. Moreau, R. Carpenter, J. How, "Distributed Spacecraft Systems Develop New GPS Capabilities," in GPS World: Formation Flight in Space Feb. 2002.

[2] W. Ren, and R. Beard, "Virtual Structure Based Spacecraft Formation Control with Formation Feedback," AIAA GNGC Conference, Aug. 2002.

[3] F. D. Busse and J. P. How, "Real-Time Experimental Demonstration of Precise Decentralized Relative Navigation for Formation-Flying Spacecraft," AIAA GNC, August 2002, Paper 2002-5003

[4] F. D. Busse and J. P. How, "Four-Vehicle Formation Flying Hardware Simulation Results," presented at the ION-GPS Conference, Sept. 2002.

[5] M. Tillerson, G. Inalhan, and J. How, "Coordination and Control of Distributed Spacecraft Systems Using Convex Optimization Techniques," International Journal of Robust and Nonlinear Control, vol 12, Issue 2-3, Feb.-Mar. 2002, p.207-242.

[6] R. Sedwick, D. Miller and E. Kong, "Mitigation of Differential Perturbations in Synthetic Apertures Comprised of Formation Flying Satellites", 9th AAS/AIAA Space Flight Mechanics Mtg, Feb. 1999.

[7] H. Schaub, K. Alfriend, " $\mathrm{J}_{2}$ Invariant Relative Orbits for Spacecraft Formations," In Goddard Flight Mechanics Symposium, May 18-20,1999, Paper No. 11.

[8] G. Inalhan, M. Tillerson, and J. How, "Relative Dynamics and Control of Spacecraft Formations in Eccentric Orbits," AIAA JGCD, vol. 25, no. 1, JanFeb 2002, p. 48-59.

[9] J. How and M. Tillerson, "Analysis of the Impact of Sensor Noise on Formation Flying Control," Proc. of the American Control Conf., June 2001, pp. 3986-3991. [10] M. Tillerson, J. How, "Advanced Guidance Algorithms for Spacecraft Formation Flying," Proceedings of American Control Conference, May 2002.

[11] Mike Tillerson, Coordination and Control of Multiple Spacecraft using Convex Optimization Techniques, S.M. Thesis, Dept. of Aeronautics and Astronautics, MIT, Jun. 2002.

[12] S. Vadali, S. Vaddi, K. Naik, K. Alfriend, "Control of Satellite Formation," AIAA GNEC Conference, Aug 2001, AIAA Paper 2001-4028. 